\title{
Modal properties of suspension electricity pylons
}

\author{
Zofia Kozyra $^{1}$, Stawomir Dudziak ${ }^{1, *}$ \\ ${ }^{1}$ Warsaw University of Technology, Faculty of Civil Engineering, al. Armii Ludowej 16, 00-637, \\ Warsaw, Poland
}

\begin{abstract}
The paper presents the results of the modal analysis of suspension electricity pylons (S12P, S24P, Y52P). The results were obtained from FEM models built in the Abaqus software. On this basis, there were proposed formulas to predict the first two natural frequencies for pylons S12P and S24P with good accuracy.
\end{abstract}

\section{Introduction}

Power lines are essential for the energetic security of each country. To increase the security of transmission a recorder has been constructed as a result (as an outcome) of the SDZP project [The System of Dynamic Management of Transmission]. The recorder monitors the deformation of the lines in real-time taking into account the electricity pylons - conductors interaction.

To determine the influence of this interaction 17 electricity pylon models were prepared by the team from the Warsaw University of Technology - the Faculty of Civil Engineering. Static and modal parameters were calculated for each model. The functions from which the first two natural frequencies can be obtained approximately for suspension pylons were proposed on the basis of the data gathered in the project.

\section{The aim of natural frequencies prediction}

In linear dynamics the dynamic response of a structure is a linear combination of eigenvectors with weights, which depend on the corresponding eigenvalues. The higher the number of the eigenvalue, the smaller the weight is in most cases (when the resonance or beat phenomenon does not occur). Therefore, the first few eigenmodes are sufficient to describe vibrations of a structure with the required accuracy [1]. Many methods of solving the linear dynamics problem use this fact because of their low numerical cost compared with the direct integration of motion equations methods [1 - 4].

For engineering applications the first natural frequency is often the most important, so many standards contain formulas to predict it. The first example is standard [5], in which the building's natural period of vibrations is associated with its height and the coefficient taking into account the type of construction. The second example is standard [6], in which

* Corresponding author: s.dudziak@il.pw.edu.pl 
the fundamental frequencies of different structures are related to their parameters such as height, mass, dimensions of their cross-sections.

The quoted examples show that engineers aim for formulas in which the prediction of the first eigenvalue is associated with the basic geometric parameters of the structure. In this paper, the formulas linking the first two natural frequencies with height for suspension towers are proposed. In paper [7] it is proved that dynamic effects cannot be omitted if a precision device is planned to be mounted on the cross arm of a pylon.

\section{Electricity pylons and their numerical models}

The paper concerns only suspension pylons, since the formulas to predict the fundamental frequency for tension towers cannot be found easily due to the variety of their design solutions, e.g. dimensions of the column projection, number of transverse ties.

Three suspension pylons of a single power line in different height versions are analysed in this paper: S12P, S24P, Y52P. All of the pylons were designed as 3D lattice frames made of steel angles with equal and non-equal sides. They consist of: columns, cross arms and arms supporting ground wires. The basic information about each pylon is summarised in Table 1.

Table 1. Characteristics of poles.

\begin{tabular}{|c|c|c|c|c|}
\hline Pylon & Height version & $\begin{array}{c}\text { Total height } \\
\text { [m] }\end{array}$ & $\begin{array}{l}\text { Arrangement } \\
\text { of conductors }\end{array}$ & Shape \\
\hline \multirow{2}{*}{$\mathrm{S} 12 \mathrm{P}$} & Basic & 22.8 & \multirow{2}{*}{ Triangular } & \multirow{2}{*}{ T-shaped } \\
\hline & +2.5 & 25.3 & & \\
\hline \multirow{4}{*}{ S24P } & -2 & 20.1 & \multirow{4}{*}{ Triangular } & \multirow{4}{*}{ T-shaped } \\
\hline & Basic & 22.1 & & \\
\hline & +5 & 27.1 & & \\
\hline & +10 & 32.1 & & \\
\hline \multirow{2}{*}{ Y52P } & -4 & 27.3 & \multirow{2}{*}{ Flat } & \multirow{2}{*}{ Y-shaped } \\
\hline & Basic & 31.3 & & \\
\hline
\end{tabular}

Pylon models were built in the Abaqus FEM software using b33 elements (EulerBernoulli beam [2-3]) as 3D frames with rigidly connected bars. The linear elastic material model of steel was assumed in the analysis. The material parameters: Young modulus $E=$ $200 \mathrm{GPa}$, Poisson's ratio $\mu=0.3$. In paper [8] the authors proved that the introduction of pinned joints in the pylon models changes the first two natural modes insignificantly. The tie rods were modelled as truss elements (symbol t3d2) made of a non-linear material which has no compressive stiffness. All the structure models have pinned supports. The models in the basic height version of each analysed pylon are shown in Figure 1. 

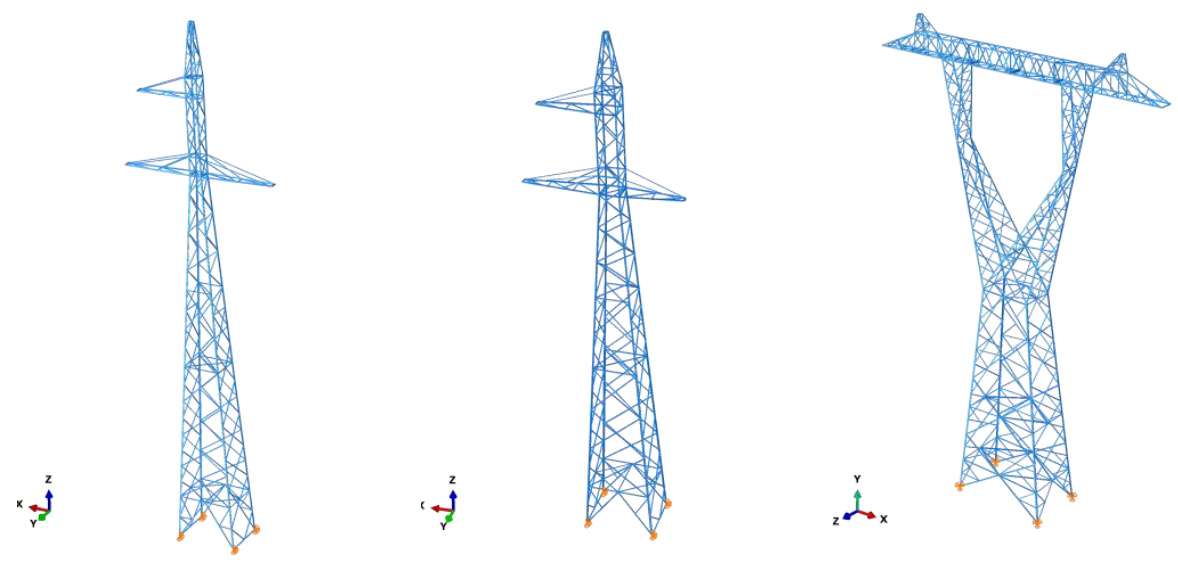

Fig. 1. The numerical models of the pylons: S12P, S24P, Y52P (left to right).

\section{Modal analysis}

Every linear FEM model used in dynamic analysis is described by two symmetric and positive definite matrices: the stiffness matrix - K and the mass matrix - M [1]. The structural eigenvalue problem consists in finding the pairs of eigenvalues - $\omega$ and eigenvectors $\mathbf{a}$. It is described by the following formula:

$$
\left(\mathbf{K}-\omega^{2} \mathbf{M}\right) \mathbf{a}=\mathbf{0} .
$$

The relation between the angular frequency $\omega$ and the normal frequency $f$ is as follows:

$$
f=\frac{\omega}{2 \pi}
$$

Some lowest eigenvalues and corresponding eigenvectors were extracted for the analyzed pylon models using the subspace iteration method implemented in the Abaqus software [2-3].

The plots which show the relation between the first three (the lowest) natural frequencies and the height of the pylons are presented in Figures 2 - 4.

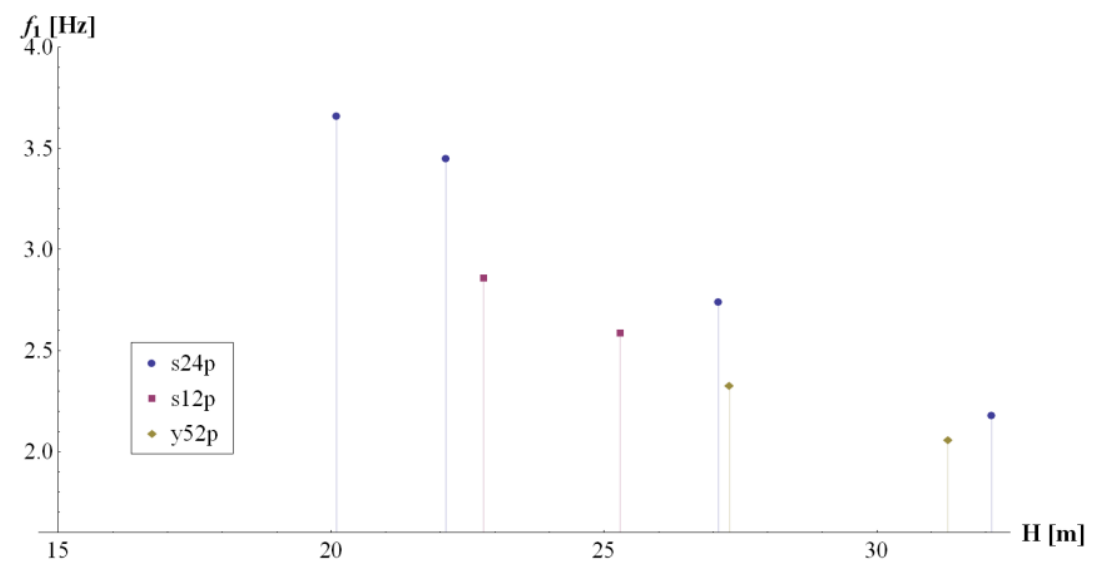

Fig. 2. The plot of relation between the first natural frequency and the height of the pylon. 


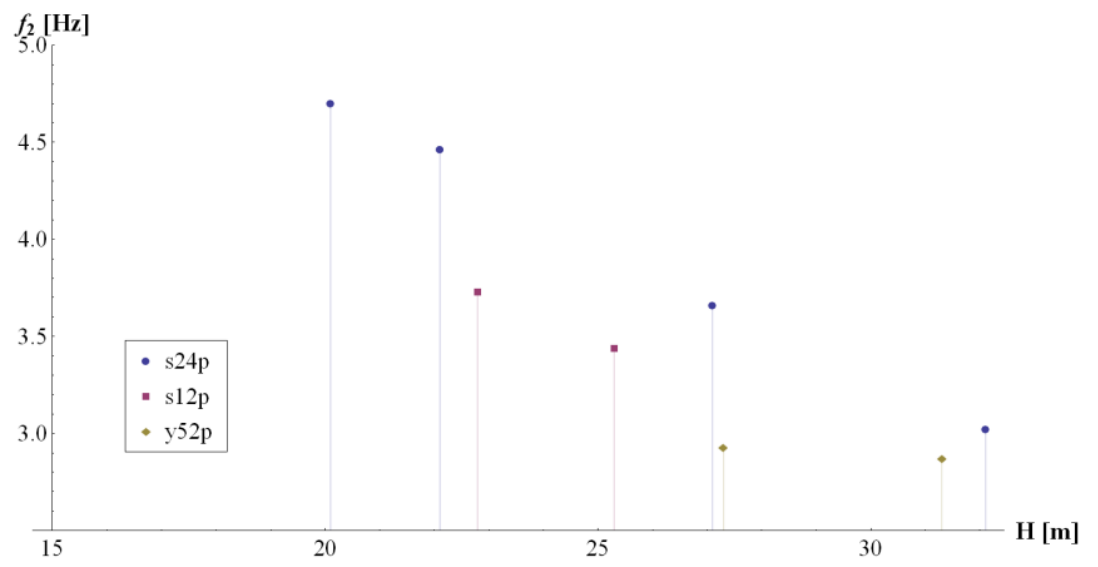

Fig. 3. The plot of relation between the second natural frequency and the height of the pylon.

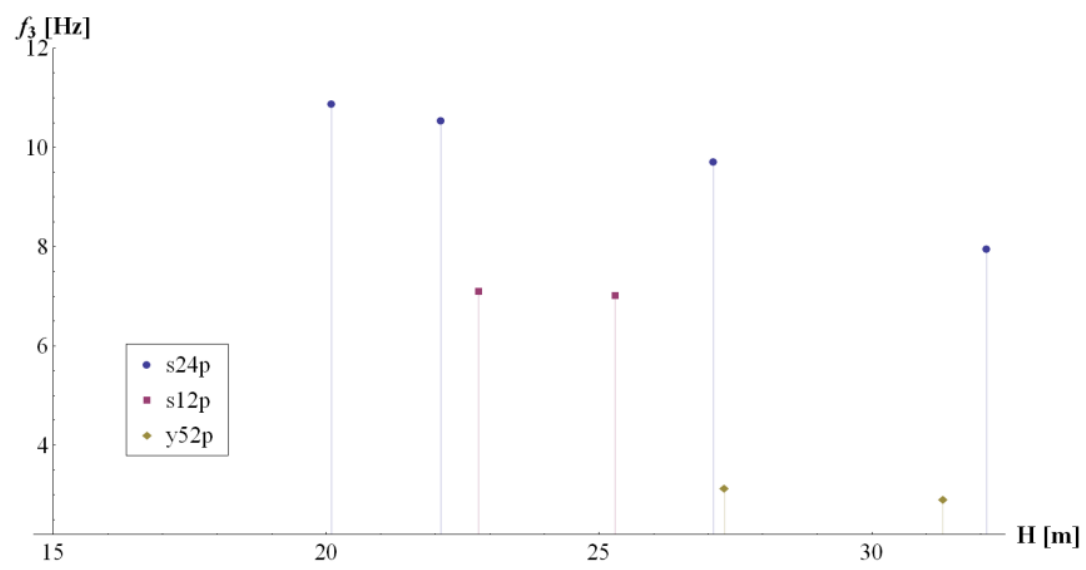

Fig. 4. The plot of relation between the second natural frequency and the height of the pylon.

Figures 5-7 present the first three eigenvectors for the basic height versions of the analysed pylons.

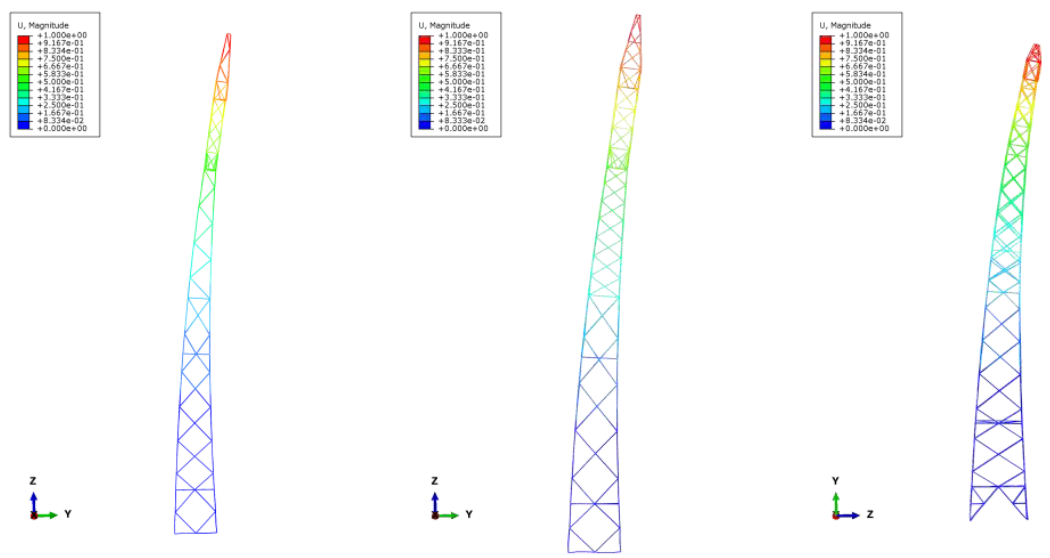

Fig. 5. The first eigenvector for the pylons: S12P, S24P, Y52P (left to right). 


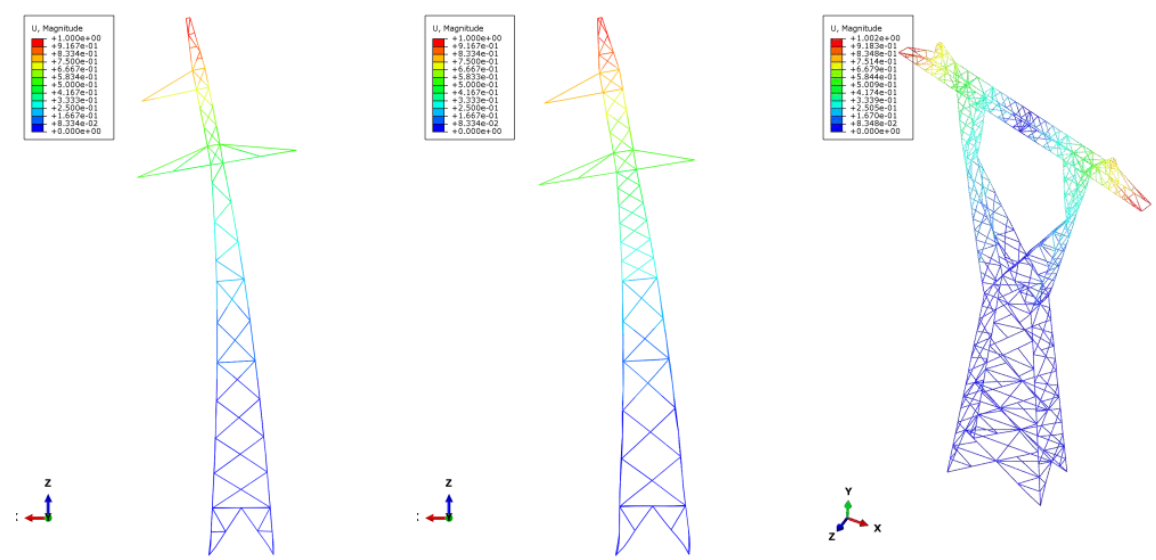

Fig. 6. The second eigenvector for the pylons: S12P, S24P, Y52P (left to right).
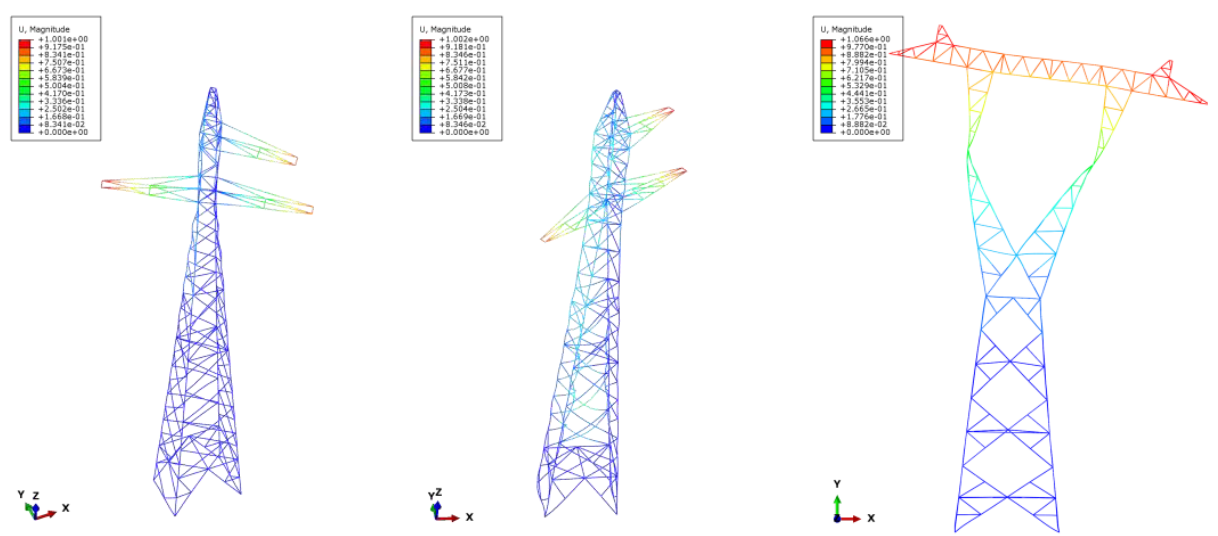

Fig. 7. The third eigenvector for the pylons: S12P, S24P, Y52P (left to right).

\section{Formulas for the approximate natural frequencies}

On the basis of the analysis of Figures $2-4$ the following conclusions can be drawn. The relation between the first two natural frequencies (numbers $i=1,2$ ) and the height $(H)$ for the S24P pylon is linear so it can be written as:

$$
f_{i}(H)=A \cdot H+B
$$

Coefficients $A$ and $B$ were found using the method of least squares. After substituting them in the formula (3) the first two natural frequencies can be approximated from the formulas:

$$
\begin{aligned}
& f_{l}(H)=-0.126 \cdot H+6.198 \\
& f_{2}(H)=-0.143 \cdot H+7.578
\end{aligned}
$$

The height $(H)$ must be substituted in metres [m] and greater than $20 \mathrm{~m}$ in the formulas above. Their results have unit $[\mathrm{Hz}]$. 
The tangent of the angle of the line drawn through the points for the S12P pylon is similar to coefficient A found for the S24P pylon. Hence, it is proposed to conduct the following algorithm for the S12P pylon:

- to substitute coefficient $A$ calculated for the S24P pylon,

- to find coefficient $B$ from the linear equation formed by assuming the height and the natural frequency for one height version,

- to compare the values for the other height version obtained from the FEM model and the proposed formula.

This approach produced the following result for the S12P pylon:

$$
\begin{aligned}
& f_{l}(H)=-0.126 \cdot H+5.732 \\
& f_{2}(H)=-0.143 \cdot H+6.990 .
\end{aligned}
$$

The results of the considerations above are shown in Figures $8-9$. The error of approximated formulas for the S12P pylon is $0.05 \mathrm{~Hz}$ for the fundamental frequency and $0.03 \mathrm{~Hz}$ for the second natural frequency.

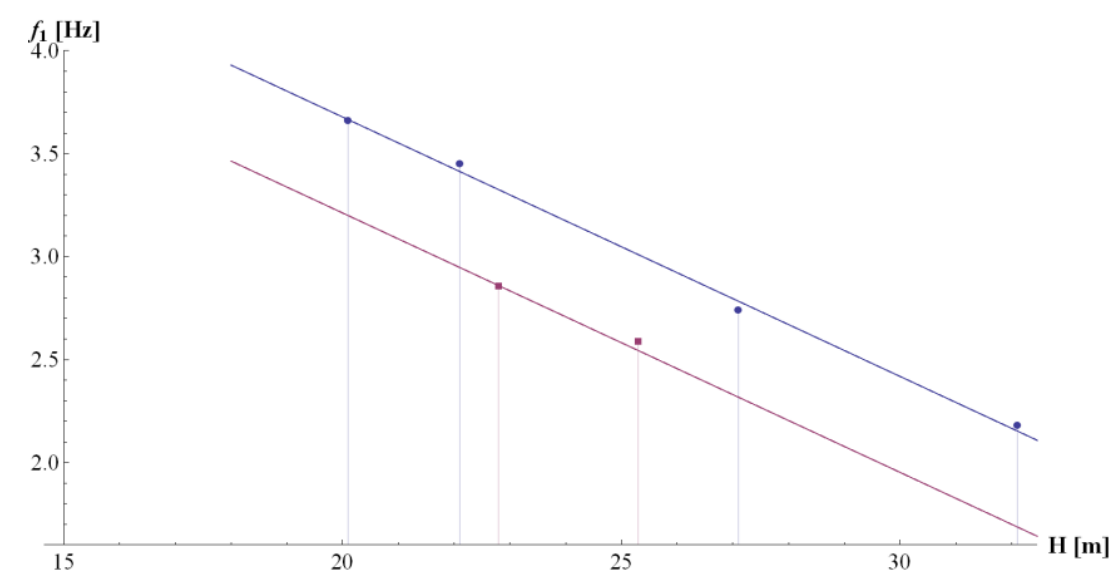

Fig. 8. The fundamental frequency for the S24P and S12P pylons obtained from FEM models and the proposed formulas.

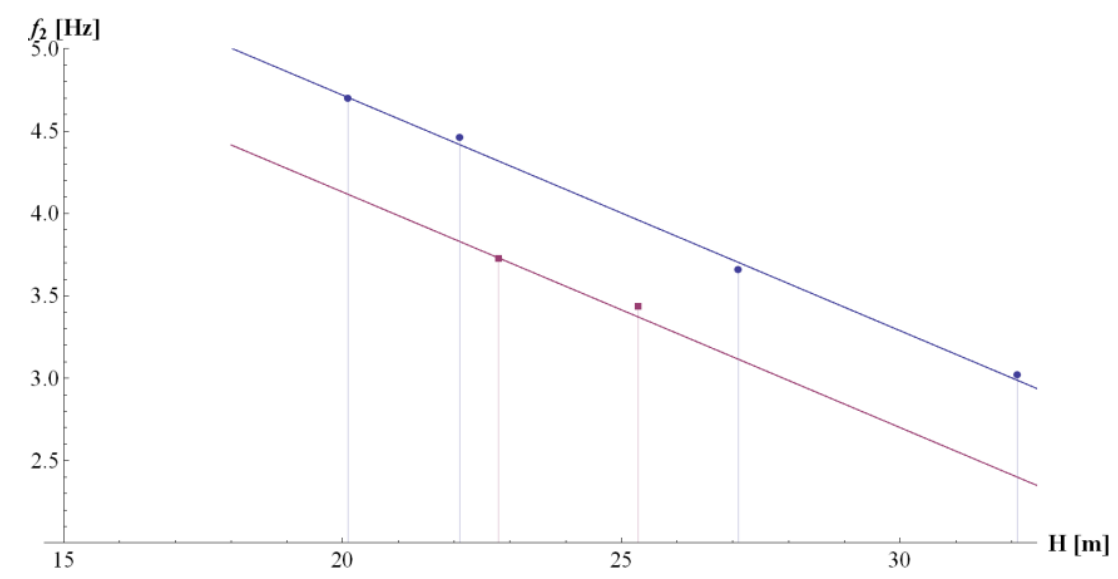

Fig. 9. The second natural frequency for the S24P and S12P pylons obtained from FEM models and the proposed formulas. 
The proposed formulas provide good estimation accuracy of the first two eigenvalues. The first two modes have bending character. On the basis of the Figure 4 analysis it can be concluded that the third eigenvalue (for the torsion mode) is not associated with the height of the pylon, but probably with the geometric properties of the cross arms.

\section{Conclusions}

On the basis of the results for two height versions of a suspension pylon, there can be found the formula to estimate the first two natural frequencies of the other versions. Good accuracy was obtained for the exemplary two towers - S12P, S24P by using the linear functions (4) - (6), which have the same directional factor.

The functions for tension towers would be more complex and have more variables. The height cannot be the only factor taken into account. The influence of properties like the cross arm span or proportions of the tower should be analysed as well.

The paper is a result of the SDZP project [System of Dynamic Management of Transmission], which was financially supported by the National Centre for Research and Development and the National Fund for Environmental Protection and Water Management in the GEKON contest (NCBR/214108).

\section{References}

1. R. Lewandowski, Dynamic of building structures (Poznań, 2006, in Polish)

2. Abaqus Analysis User's Manual (Dassault Systèmes, 2012)

3. Abaqus Theory Manual (Dassault Systèmes, 2012)

4. K.J. Bathe, Finite element procedures (Prentice Hall, 1996)

5. EN 1998-1 Eurocode 8: Design of structures for earthquake resistance - Part 1 : General rules, seismic actions and rules for buildings

6. EN 1991-1-4 Eurocode 1: Actions on structures - Part 1-4: General actions - Wind actions

7. S. Dudziak, Z. Kozyra, TTS 12, 2463-2467 (2015)

8. S. Dudziak, Z. Kozyra, Theoretical Foundations of Civil Engineering, vol. 23, W. Szcześniak, ed., 79-88 (2015) 